
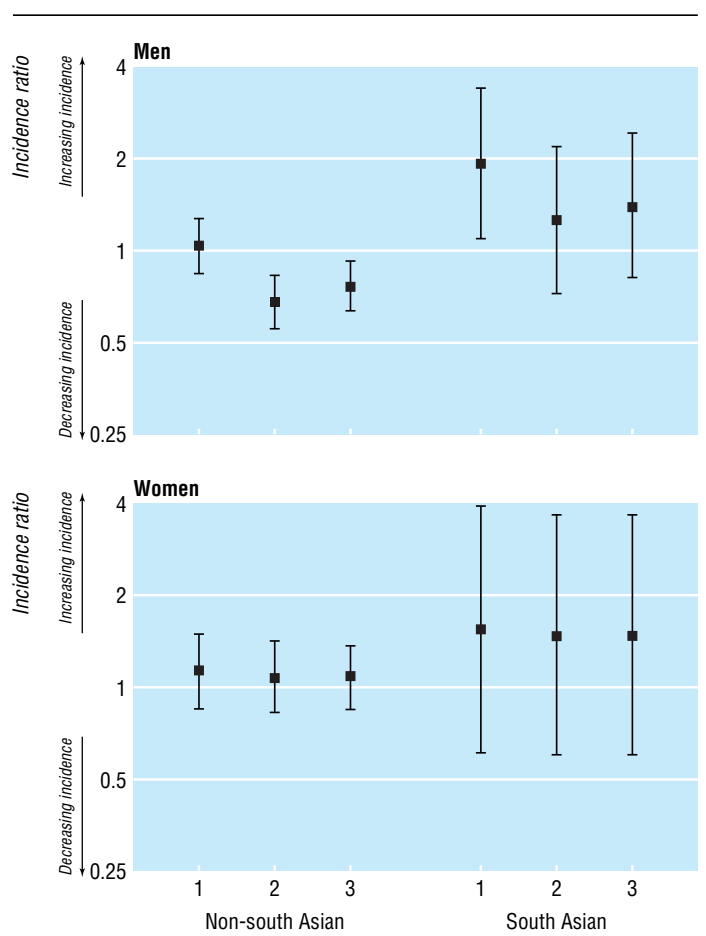

Deprivation tertile (1=least deprived, $3=$ most deprived)

Incidence ratios (95\% confidence intervals) of lung cancer comparing incidence for 1995-9 with that for 1990-4 among south Asian and non-south Asian men and women by deprivation tertile (1=least deprived, $3=$ most deprived). Values (log scale) from Poisson regression models including interactions between time period and deprivation tertile and between time period and ethnicity

\section{Comment}

Our findings confirm lower rates of lung cancer for south Asian men than non-south Asian men but suggest differing trends, with incidence increasing among south Asian men but falling among non-south Asians. There is a tendency to emphasise the importance of cancers such as those of the head and neck among south Asians because they are relatively more common than in the majority UK population. However, Bhopal and Rankin also highlight the need to look at absolute numbers of cancer cases, ${ }^{2}$ which show that lung cancer is the commonest cancer for both south Asian and non-south Asian men. With smoking rates higher among UK south Asians aged 30-49 years than those aged 50-74, ${ }^{4}$ lung cancer prevention is a high priority. Health promotion programmes should target the whole population, and the tendency to dismiss south Asians as "low risk" populations for smoking related disease needs to be revised.

We thank Louise Hollingworth for her help in providing data from the Trent Cancer Registry. Material from Crown Copyright records was made available through the Post Office and the ESRC data archive. We thank Hilda Parker for her comments on the manuscript, and we appreciate the advice of Albert Benghiat, lead clinician for Leicestershire Cancer Services Network.

Contributors: LKS and JLB contributed to the conception and design of the study and secured its funding. LKS analysed the data, and all three authors interpreted the results and wrote the paper. LKS is the guarantor for the study.

Funding: LKS is funded to undertake the research as an MRC/ Trent Region training fellow in health services research.

Competing interests: None declared.

1 Winter H, Cheng KK, Cummins C, Maric R, Silcocks P, Varghese $C$. Cancer incidence in the south Asian population of England (1990-92). BrJ Cancer 1999;79:645-54

2 Bhopal RS, Rankin J. Cancer in minority ethnic populations: priorities from epidemiological data. Br J Cancer 1996;29:S22-32.

3 Cummins C, Winter H, Cheng KK, Maric R, Silcocks P, Varghese C. An assessment of the Nam Pehchan computer program for the identification of names of south Asian ethnic origin. J Public Health Med 1999:21:401-6.

4 Johnson M. Black and minority ethnic groups in England: the second health and lifestyles survey. London: Health Education Authority, 1999.

(Accepted 24 June 2002)

\title{
RESEARCH POINTERS
}

\section{Platelet responsiveness to aspirin in patients with hyperlipidaemia}

Maribeth Friend, Ivana Vucenik, Michael Miller

Platelet responsiveness to aspirin is reduced in patients with hyperlipidaemia

continued over
Aspirin $325 \mathrm{mg}$ /day reduces the rate of events associated with coronary heart disease. In most people, aspirin produces irreversible inhibition of platelet aggregation, but in a sizeable minority of patients, the degree of platelet aggregation needed to prevent events according to in vitro assessments is not achieved. ${ }^{1}$ Risk factors for coronary heart disease may contribute to aspirin resistance (the inability of aspirin to protect individuals from thrombotic complications), so aspirin may not be cardioprotective in patients with hyperlipidaemia. ${ }^{2}$ We evaluated patients with a range of cholesterol concentrations to determine the impact of hypercholesterolaemia on platelet responsiveness in patients treated with aspirin.

\section{Participants, methods, and results}

Consecutive patients $(n=56)$ were recruited from the University of Maryland Preventive Cardiology

Outpatient Center. The mean (SD) age was 54.3 (11.1) 
years, and $40(72 \%)$ of the patients were men. Patients were eligible if they were taking aspirin $325 \mathrm{mg}$ /day and presented with a history of coronary heart disease or at least two risk factors for coronary heart disease. Patients were excluded if they used heparin, warfarin, or other antiplatelet agents or had consumed ethanol within 96 hours of enrolment. The study was approved by the university's institutional review board.

Whole blood was drawn into tubes containing $3.2 \%$ buffered sodium citrate and was tested within four hours. Platelet aggregation was assessed by electrical impedance with a dual sample aggregometer and a final concentration of collagen of $1.0 \mu \mathrm{g} / \mathrm{ml}^{3}{ }^{3}$ Fasting concentrations of total cholesterol and triglycerides in plasma were measured with commercial kits and an automatic chemistry analyser. Concentrations of high density lipoprotein (HDL) cholesterol were measured after apolipoprotein B-containing lipoproteins were precipitated out. The concentration of low density lipoprotein (LDL) cholesterol was calculated by subtracting the sum of the concentration of HDL cholesterol and one fifth of the concentration of triglycerides from the total cholesterol concentration. Hyperlipidaemia was defined as a total cholesterol concentration $>6.2 \mathrm{mmol} / \mathrm{l}$. Poor platelet responsiveness to aspirin was defined as aggregation of $\geqslant 50 \%$ of platelets and represented the top quarter of samples.

The 14 patients with poor responsiveness to aspirin had significantly higher mean (SD) concentrations of total cholesterol and LDL cholesterol, respectively, than the 42 patients with good responsiveness (6.2 (1.6) vs 4.8 (1.2), $\mathrm{P}=0.004$, and 4.0 (1.7) vs 3.0 (1.1), $\mathrm{P}=0.03)$. In total, $9 / 13(69 \%)$ patients with hyperlipidaemia had poor responsiveness to aspirin. No significant differences in concentrations of HDL cholesterol and triglycerides, history of cigarette smoking, or prevalence of hypertension or diabetes mellitus were seen between those with poor and good responsiveness to aspirin. Interestingly, 12/14 (86\%) patients with poor responsiveness to aspirin were taking lipid lowering therapy.

Significant correlations were seen between the degree of platelet aggregation produced by $1 \mu \mathrm{g} / \mathrm{ml}$ of collagen and the concentrations of total cholesterol $(\mathrm{r}=0.35, \mathrm{P}=0.009)$ (figure) and of triglycerides $(\mathrm{P}=0.049)$. Correlations were not seen with concentrations of HDL cholesterol or LDL cholesterol; the lack of correlation with LDL cholesterol may have been, in part, because LDL cholesterol concentrations could not be estimated for four patients with hypertriglyceridaemia (triglycerides $>4.5 \mathrm{mmol} / \mathrm{l})$.

We compared platelet aggregation in patients in the top and bottom quarters of total cholesterol concentrations. Patients with total cholesterol concentrations $<4.14 \mathrm{mmol} / 1$ (bottom quarter) produced less platelet aggregation, and thus had better platelet responsiveness to aspirin, than patients in the upper quarter of cholesterol concentrations ( $7 \%$ v $64 \% ; \mathrm{P}=0.004$ by $\chi^{2}$ analysis).

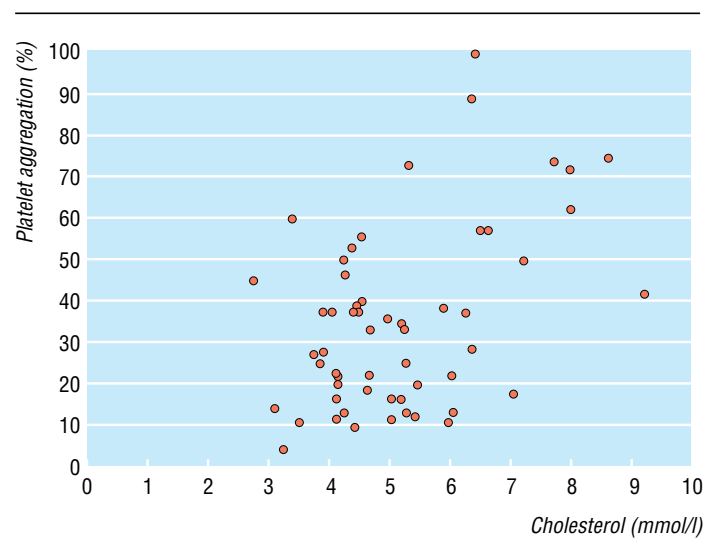

Scattergram of platelet aggregation and total cholesterol concentration

\section{Comment}

Despite the cardiovascular benefits of cholesterol lowering treatments, morbidity and mortality from coronary heart disease are unacceptably high. Platelets taken from hyperlipidaemic patients are highly thrombogenic, but other factors, such as upregulation of cyclooxygenase-2 expression, may contribute to poor platelet responsiveness to aspirin. ${ }^{25}$ Patients who respond poorly to aspirin may need higher doses of aspirin, alternative antiplatelet agents (such as clopidogrel), or further reductions in concentrations of total cholesterol and LDL cholesterol.

Contributors: MF, IV, and MM designed the study and wrote the paper. MF and MM did the analysis. IV and MM edited the paper. IV and MM are guarantors for the paper.

Funding: Supported in part by NIH grant (HL 61369) and a Veteran's Affairs Merit Award to MM.

Competing interests: None declared.

1 Helgason CM, Bolin KM, Hoff JA, Winkler SR, Mangat A, Tortorice KL, et al. Development of aspirin resistance in persons with previous ischemic stroke. Stroke 1994;25:2331-6.

2 Carvalho ACA, Colman RW, Lees RS. Platelet function in hyperlipoproteinemia. N Engl J Med 1974;290:434-8.

3 Sathiropas P, Marbet GA, Sahaphong S, Duckert F. Detection of smal inhibitory effects of acetylsalicylic acid by platelet impedance aggregometry in whole blood. Thromb Res 1988;51:55-62.

4 Kawasaki T, Ozeki Y, Igawa T, Kambayashi J. Increased platelet sensitivity to collagen in individuals resistant to low-dose aspirin. Stroke 2000;31:591-5.

5 Weber A-A, Zimmermann K, Meyer-Kirchrath J. Cyclooxygenase-2 in human platelets as a possible factor in aspirin resistance. Lancet 1999;353:900.

(Accepted 24 June 2002)

\section{Endpiece}

\section{A necessity}

As you get older it is harder to have heroes, but it is sort of necessary.

Ernest Hemingway in The Old Man and the Sea, which he wrote in 1952 when he was 53

Submitted by Fred Charatan,

retired geriatric physician, Florida
Department of

Medical and

Research

Technology and

Medicine,

University of

Maryland Medical

Center, Baltimore,

MD 21201, USA

Maribeth Friend

graduate student

Ivana Vucenik

associate professor

Division of

Cardiology,

University of

Maryland Medical

Center

Michael Miller

associate professor of

medicine and

epidemiology

Correspondence to:

M Miller

mmiller@heart.

umaryland.edu 